\title{
PERIODIC GW LEVEL MICROWAVE PULSES IN X-BAND FROM A COMBINATION OF A RELATIVISTIC BACKWARD WAVE OSCILLATOR AND A HELICAL WAVEGUIDE COMPRESSOR*
}

\author{
P. MacInnes ${ }^{1}$, V.L. Bratman ${ }^{2}$, L. Zhang ${ }^{1}$, G.G. Denisov ${ }^{2}$, W. He ${ }^{1}$, N.G. Kolganov ${ }^{2}$, \\ M. McStravick ${ }^{1}$, S.V. Mishakin ${ }^{2}$, C.W. Robertson ${ }^{1}$, S.V. Samsonov ${ }^{2}$, C.G. Whyte ${ }^{1}$, \\ A.R. Young ${ }^{1}$, K. Ronald ${ }^{1}$, A.D.R. Phelps ${ }^{1}$ and A.W. Cross ${ }^{1}$ \\ ${ }^{I}$ Department of Physics, SUPA, University of Strathclyde, 107 Rottenrow, Glasgow, G4 ONG, Scotland, UK \\ ${ }^{2}$ Institute of Applied Physics, Russian Academy of Sciences, Nizhny Novgorod, 603950, Russia.
}

\begin{abstract}
Backward Wave Oscillators (BWO's) utilizing moderately relativistic $(\sim 550 \mathrm{kV})$, high-current $(\sim 10 \mathrm{kA})$ electron beams are capable of producing hundreds of MWs of pulsed radiation in the centimeter wavelength range. Such relativistic BWOs (RBWOs) allow for broadband, smooth, frequency-tuning via adjustment of the accelerating potential; making them an attractive source for use in frequency-swept pulse compression.

This paper presents results of a $2.86 \mathrm{~m}$ long 5 -fold helically corrugated, dispersive pulse compressor where a maximum power compression ratio of 25 was achieved by compressing an input microwave pulse of 80 ns duration swept from $9.65 \mathrm{GHz}$ to $9.05 \mathrm{GHz}$ into a $1.6 \mathrm{~ns}$ Gaussianenvelope pulse. For an average input power of $5.8 \mathrm{~kW}$ generated by a conventional traveling wave tube amplifier, a peak pulse output power of $145 \mathrm{~kW}$ was measured corresponding to an energy efficiency of $66 \%$.

An X-band relativistic BWO, designed to drive a $0.97 \mathrm{~m}$ long 5-fold compressor, was built and tested using the accelerating potential generated by a SINUS- 6 highcurrent accelerator. The experimental RBWO operated close to the predicted power of $700 \mathrm{MW}$ with its oscillation frequency varied from 10 to $9.6 \mathrm{GHz}$ via the falling edge of the voltage pulse. It was demonstrated that the $\sim 15 \mathrm{~ns}$ duration frequency-swept part of the RBWO pulse was effectively compressed resulting in a 4.5 -fold peak power increase with a maximum power of $3.2 \mathrm{GW}$ generated. The potential for a 5 -fold helical waveguide to compress longer duration pulses generated by a RBWO is discussed.
\end{abstract}

\section{I.INTRODUCTION}

Pulse compression technology that converts long duration, low power pulses into short, high peak power pulses is commonly used in applications that require high peak power and pulsed operation such as radar systems
[1]. Gigawatt level microwave radiation can be achieved by a frequency-swept multi-megawatt pulse generated by a high power vacuum electronic device acting as the input source for a microwave pulse compressor based on a high fold helically corrugated waveguide (HCW) [2]. Such gigawatt level signals are otherwise extremely difficult to realise.

The use of a metal waveguide as a dispersive medium to generate high peak power microwaves is a well-known technique of compression. The principle is to use a sweptfrequency-modulated pulse train with a monotonically increasing group velocity propagating through the waveguide. The tail of the pulse will overtake its leading edge which results in pulse shortening and growth in power amplitude if the losses are small.

A smooth waveguide can have a relatively large monotonic group velocity difference over a wide frequency range. From the dispersion curve of the smooth waveguide, the group velocity has a large change near the cutoff frequency (shown as $\omega_{1}$ and $\omega_{2}$ in Figure 1) whilst the group velocity changes less at frequencies further away from the cutoff frequency. Therefore most of the frequency range suitable for the pulse compression is near the cutoff. However, the large Ohmic loss close to cutoff results in reduced energy efficiency giving relatively low compression ratios being achieved as evidenced by a compression ratio of 7 measured in an experiment using a $19.2 \mathrm{~mm}$ diameter smooth $6.6 \mathrm{~m}$ long circular waveguide at X band [3]. The helically corrugated waveguide (HCW) studied in this paper demonstrates much better performance than the smooth waveguide. The crosssectional and longitudinal periodicities of a $\mathrm{HCW}$ creates operating eigen modes ( $W_{1}$ and $W_{2}$ ) whose properties can be represented by resonant coupling of two modes (1 and 2) which are independent solutions in circular waveguide, as shown in Figure 1. Around the coupling point $P_{0}$, the group velocity of operating mode $W_{1}$ is monotonically decreasing over a relatively wide frequency band as

\footnotetext{
* Work was supported by the EPSRC UK (research grant EP/K029746/1) and the Russian Foundation for Basic Research (Grant 10-02-00975). The EPSRC sponsorship of the research studentship of M. McStravick is gratefully acknowledged.
} 
frequency increases. $W_{1}$ also has a low Ohmic loss at this frequency band as it is far from its cutoff. A further advantage of the $\mathrm{HCW}$ is that because the rapid change in group velocity is achieved using an inflexion in the dispersion curve. If a compressor operated at near cutoff frequency then a large reflection could occur due to a small difference in the waveguide diameter as a consequence of the tolerance achieved during machining. The reflection may cause feedback oscillations and possible damage to driving amplifier or oscillator. The use of the HCW can mitigate such risks.

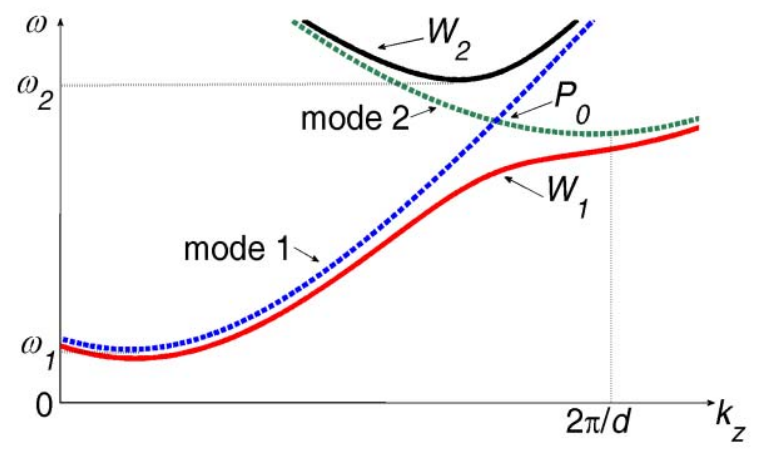

Figure 1 The mode coupling in the HCW. The eigenmodes $W_{1}$ and $W_{2}$ are the result of the coupling of modes 1 and 2 of a circular waveguide. The group velocity of operating mode $\mathrm{W}_{1}$ is monotonically decreasing over a relatively wide frequency band as frequency increases around the coupling point $\left(\mathrm{P}_{0}\right)$.

In this paper, the pulse compression experiment using a five-fold $\mathrm{HCW}$ with an average diameter of $65.7 \mathrm{~mm}$ for enhanced power handling is presented. The power handling capability of the five-fold HCW for use with a relativistic Backward Wave Oscillator (RBWO) is also presented.

\section{II.RESULTS}

\section{A. Experimental results 5-fold compressor (2.86m long)}

The HCW was designed to operate with a high order mode to achieve the coupling of the $1^{\text {st }}$ space harmonic $\mathrm{TE}_{31}$ mode with the $\mathrm{TE}_{22}$ mode, which determined a fold number of 5 for the HCW from the azimuthal synchronism condition $\mathrm{m}_{2}-\mathrm{m}_{1}=\mathrm{m}_{\mathrm{B}}$ to be satisfied, where $\mathrm{m}_{1}$ and $\mathrm{m}_{2}$ are the azimuthal indices of the two coupling modes and $\mathrm{m}_{\mathrm{B}}$ is the azimuthal period of the waveguide. $m_{2}=2$ and $m_{1}=-3$ indicates an opposite rotation of the $\mathrm{TE}_{31}$ mode. The dispersion characteristic can be changed by varying the HCW's geometry whose profile is governed by the expression

$$
\mathrm{r}(\varphi, \mathrm{z})=\mathrm{R}_{0}+\mathrm{R}_{1} \cos (5 \varphi+2 \pi \mathrm{z} / \mathrm{d})
$$

in a cylindrical coordinate system $(r, \varphi, z)$. The mean radius $\mathrm{R}_{0}$ mainly decides the operating frequency range; the corrugated depth $\mathrm{R}_{1}$ mostly controls the coupling strength, and the period d directly affects the position of the coupling point. The optimal dimensions used in the experiments were derived to be a five-fold HCW with $\mathrm{R}_{0}=32.84 \mathrm{~mm}, \mathrm{R}_{1}=2.43 \mathrm{~mm}$, and $\mathrm{d}=33.26 \mathrm{~mm}$. The total length of $2.86 \mathrm{~m}$ was decided by the optimum trade-off between compression ratio and energy efficiency.

The rectangular profile input pulse used in the experiment was desired for the experiment. Its frequency evolution was calculated from the measured dispersion curve of the whole device. The starting frequency was 9.6 $\mathrm{GHz}$, which corresponds to the minimum group velocity (about $0.15 \mathrm{c}$, where $\mathrm{c}$ is the speed of light) in the compressor. To generate the $0.6 \mathrm{GHz}$ bandwidth frequency swept signal as the input, an arbitrary waveform generator (AWG, model: Agilent N6030A) which is capable of generating a $\pm 500 \mathrm{MHz}$ modulated signal was chosen and programmed. The $\pm 300 \mathrm{MHz}$ frequency sweeping signal was then mixed with a 9.35 $\mathrm{GHz}$ oscillation signal by a vector signal generator (VSG, model: Agilent E8257D) to generate the frequency sweeping signal at the frequency range from $9.65 \mathrm{GHz}$ to $9.05 \mathrm{GHz}$ for pulse compression. To demonstrate the experiment at multiple kilowatts power level, a conventional X-band travelling wave tube (TWT) from TMD Ltd. was used at the University of Strathclyde to amplify the low power input signal to a few kWs. The signals from the TWT and the output compressed pulses were recorded by a $20 \mathrm{GHz}$ digitizing storage oscilloscope (DSO, model Agilent DSOX92004A) which was protected with the use of a $-10 \mathrm{~dB}$ coupler with additional $-50 \mathrm{~dB}$ attenuation. The setup of the pulse compression experiment is shown in Figure 2.

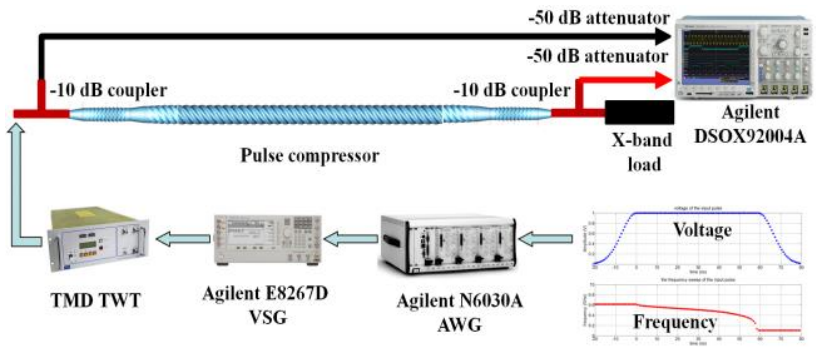

Figure 2 The schematic of the microwave pulse compression experimental setup.

The phase information was extracted from the experimentally measured real time microwave signals measured using the $20 \mathrm{GHz}$ DSO. The power of the signals was then calculated from the measured voltages. The power compression factors for the three cases are 22.2, 21.5 and 21.9 respectively, and agree well with each other. The input signal and the compressed output signal are shown as Figure 3.

The best compression factor of 25.2 was obtained using a frequency sweep over an 80 ns part of the input pulse with roughly constant amplitude figure 3 , corresponding to an average power of about $5.80 \mathrm{~kW}$. The peak power of the compressed pulse was $144.8 \mathrm{~kW}$ with a pulse width of $1.6 \mathrm{~ns}$ as shown in Figure 4 resulting in an energy efficiency of $66 \%$ [4]. 


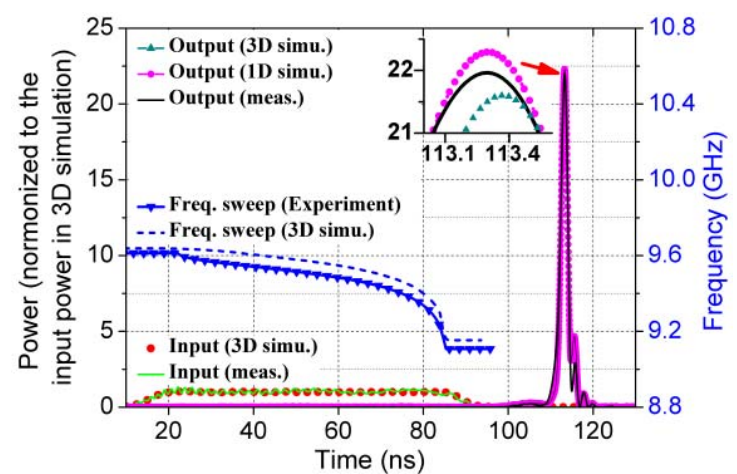

Figure 3 The simulated and measured compressed pulses. The detail of the compression factors for the different cases are shown in the inset.

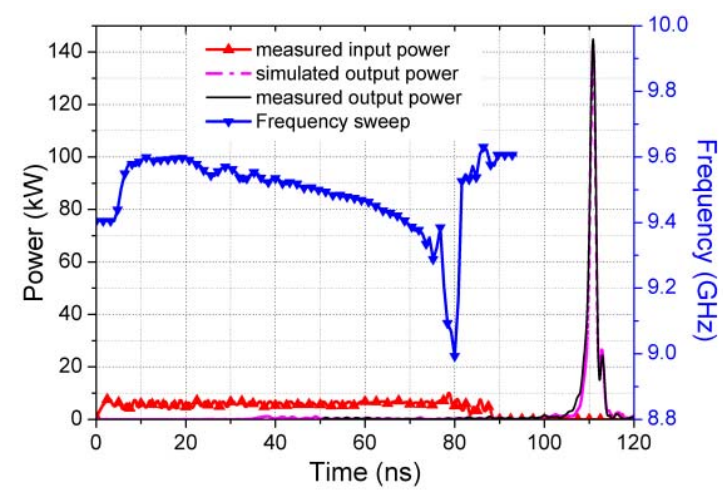

Figure 4 The measured and simulated microwave output power from the compression experiment, the input microwave waveform and its frequency sweep.

\section{B. Experimental results of 5-fold compressor $(0.97 \mathrm{~m})$}

Pulse compression experiments conducted at IAP/RAS in which multigigawatt peak power X-band radiation was generated using a relativistic BWO (RBWO) powered by a SINUS-6 high-current accelerator driving a $0.97 \mathrm{~m}$ long 5 -fold helically corrugated waveguide. A schematic of the experiment is shown in figure 5 .

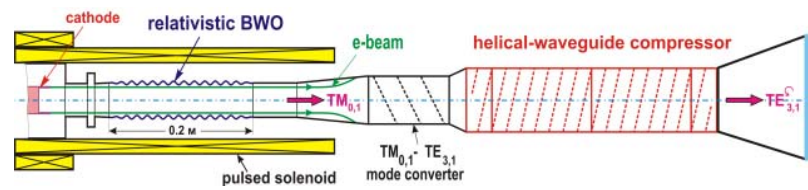

Figure 5 Scheme of the experimental setup

In the experiment a five-fold sinusoidal corrugation similar to that described in section $\boldsymbol{A}$. These relatively high-order modes ensured sufficient RF breakdown strength at gigawatt power level. The inner surface of the waveguide in cylindrical coordinates $\mathrm{r}, \varphi, \mathrm{z}$ had the following form:

$$
r(\varphi, z)=31.5+2.7 \sin (2 \pi z / 32.4-5 \varphi)
$$

(all dimensions in millimeters). The pulse compressor consisted of 4 aluminum sections and had a total length of regular corrugation of $972 \mathrm{~mm}$, bounded on each side by three-period sections with linearly increasing/decreasing amplitude of corrugation for adiabatic conversion.
The compressor performance can be determined by comparing the strength of the $\mathrm{TE}_{3,1}$ modes at the input and output from the experiment. The most favorable frequency region for pulse compression is a part of the dispersion characteristic where the operating wave group velocity has a large negative gradient as a function of frequency, which, for the HCW under consideration, is the region from 9.5 to $10 \mathrm{GHz}$ (Figure.6). Therefore, in order to be effectively compressed, an input pulse should have a negative frequency sweep within this frequency interval.

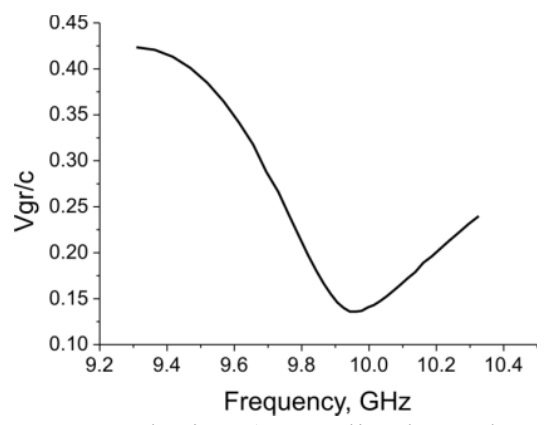

Figure 6 Group velocity (normalized to the speed of light) of the $\mathrm{HCW}$ operating wave

An RBWO which combines high output power, high efficiency with the ability to be frequency tuned over a sufficiently wide range by a moderately relativistic electron beam was designed. According to simulations carried out using the time-domain PIC code KARAT, the RBWO oscillation frequency should smoothly sweep from 10 to $9.5 \mathrm{GHz}$ when the accelerating voltage gradually changed in time from 600 to $300 \mathrm{kV}$

The experiments were performed using a SINUS-6 accelerator (IAP RAS) where an accelerating voltage pulse was formed by a combination of a Tesla transformer and a coaxial transmission line. When operating into a matched load it produces pulses of voltage up to $600 \mathrm{kV}$ and current of 6-7 kA with about 15 -ns long flat top and 5 to 10 -ns long rising/falling edges. Because of the principle of operation of the SINUS-6 accelerator it is problematic to realize the necessary voltage sweep for the RBWO voltage over the main central region of the pulse by modifying the external circuit. Nevertheless, a technique was experimentally established to realize an appropriate voltage droop "internally". To achieve this change in voltage (Figure 7a) a dielectric cylinder was introduced into the region of the magnetically insulated coaxial diode where the electron beam was formed.

By varying the axial distance between the edges of the cathode and dielectric insert by several millimeters the magnitude of the voltage drop and hence the frequency sweep interval could be controlled. This capability was used in the experimental optimization of the combination of the RBWO with the compressor. At optimum configuration, when the voltage decreased from 550 to $300 \mathrm{kV}$ whilst the beam current increased from 8 to $10 \mathrm{kA}$ the RBWO generated radiation with power of $0.6-0.8 \mathrm{GW}$ 
when frequency sweeping from 10 to $9.6 \mathrm{GHz}$ over a period of about $15 \mathrm{~ns}$ (Figure 7b).
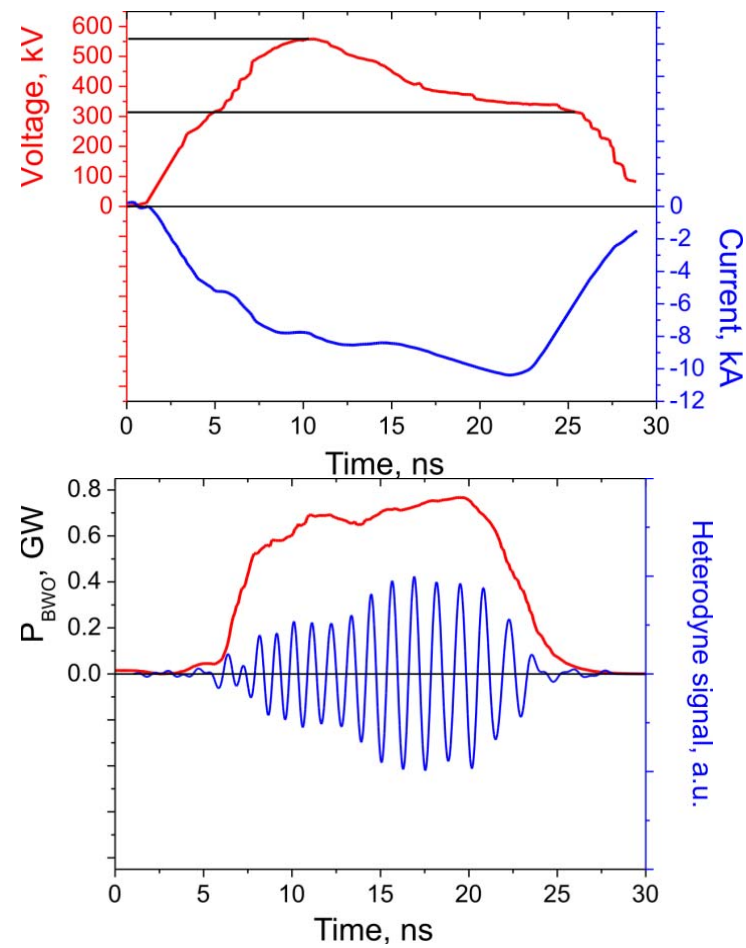

Figure 7 Temporal evolution of the RBWO: a) accelerating voltage (red curve) and beam current (blue curve); b) output power (red curve) and heterodyne signal (blue curve) for a master oscillator frequency of $9.1 \mathrm{GHz}$.

The frequency sweep was measured by means of a heterodyne technique, when a small fraction of output radiation from the RBWO was non-linearly mixed with radiation from a pulsed magnetron at a fixed frequency, resulting in a waveform at the intermediate frequency (Fig. 7b, blue curve). The power of the output RF pulse (Fig. 7b, red curve) from the RBWO was obtained by processing the RF detector signal via the detector voltagepower calibration and data from a calorimeter measuring the total RF energy in the pulse.

The RBWO was combined with the HCW compressor in a common vacuum system via a mode converter which transformed the power from the RBWO in the form of the $\mathrm{TM}_{0,1}$ mode into the incident operating mode of the compressor, namely, the circularly polarized $\mathrm{TE}_{3,1}$ mode (Figure 5).

The experimental optimization aiming to obtain the maximum peak power of the compressed pulse was performed by variation of the axial distance between the cathode and dielectric insert, electron beam diameter (factors influencing the RBWO power and frequency sweep) and the length of the HCW which could be adjusted in 6-period-long sections. As a result the maximum peak power of the compressed pulse was measured to be as high as $3.2 \mathrm{GW}$ with a halfwidth of $2.2 \mathrm{~ns}$ with a total pulse energy of $9.7 \pm 0.2 \mathrm{~J}$ (Figure 8). As compared to the RBWO having approximately $0.7 \mathrm{GW}$ pulse-averaged output power and $10.2 \pm 0.2 \mathrm{~J}$ of pulse energy, a 4.5 -fold peak power amplification due to the compression was achieved. Assuming the energy content in a "useful" pulse as a product of its peak power and halfwidth a compressing efficiency of nearly $70 \%$ was realized. (the rest of energy goes to ohmic losses and the pulse sidelobes).

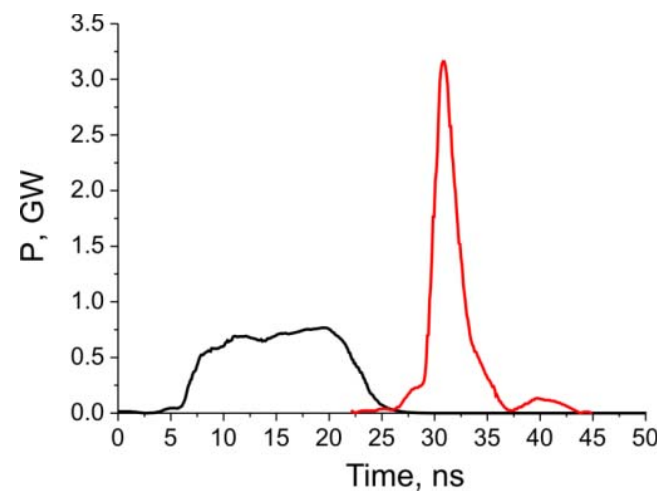

Figure 8 The resultant compressed pulse (red curve) shown in contrast to the RBWO output pulse (black curve).

\section{III.SUMMARY}

It should be noted that the achievable power compression ratio is approximately proportional to the input frequency modulated pulse width. Therefore if the duration of the decreasing RBWO accelerating voltage pulse may be extended from $15 \mathrm{~ns}$ to $80 \mathrm{~ns}$ and the length of the helical waveguide compressor is proportionally elongated, as demonstrated in this paper, a further power increase beyond the $3.2 \mathrm{GW}$ compressed pulse achieved in this work is possible

\section{REFERENCES}

[1] A. V. Gaponov-Grekhov and V. L. Granatstein, Applications of High-Power Microwaves, Boston, Artech House, 1994.

[2] V. L. Bratman, G. G. Denisov, N. G. Kolganov, S. V. Mishakin, S. V. Samsonov, A. W. Cross, W. He, L. Zhang, M. McStravick, C. G. Whyte, A. R. Young, K. Ronald, C. W. Robertson, and A. D. R. Phelps, "Generation of $3 \mathrm{GW}$ microwave pulses in X-band from a combination of a relativistic backward-wave oscillator and a helical-waveguide compressor," Phys. Plasmas, vol. 17, pp. 110703, 2010.

[3] S. V. Samsonov, A. D. R. Phelps, V. L. Bratman, G. Burt, G. G. Denisov, A. W. Cross, K. Ronald, W. He, and H. Yin, "Compression of Frequency-Modulated Pulses using Helically Corrugated Waveguides and Its Potential for Generating Multigigawatt RF Radiation," Phys. Rev. Lett., vol. 92, no. 11, pp. 118301, Mar. 2004.

[4] L. Zhang, S.V. Mishakin, W. He, et al, "Experimental Study of Microwave Pulse Compression Using a FiveFold Helically Corrugated Waveguide", IEEE Trans on Micro. Theory and Tech., 63, pp1090-1096, 2015. 